\title{
Using Finite Element Analysis and Experimental Analysis on Vibration of a Piezoelectric Micro Pump
}

\author{
Bo-Wun Huang1, Jung-Ge Tseng', Chien-Hua Chang1, Chen-Yuan Li ${ }^{3}$ \\ ${ }^{1}$ Graduate Institute of Mechatronics Engineering, Cheng Shiu University, Kaohsiung, Taiwan \\ ${ }^{2}$ Medical Mechatronics Engineering Program, Cheng Shiu University, Kaohsiung, Taiwan \\ ${ }^{3}$ Department of Mechanical Engineering, Chinese Culture University, Taipei, Taiwan \\ Email: huangbw@gcloud.csu.edu.tw, james.tseng@gcloud.csu.edu.tw, master.ca5302@msa.hinet.net, \\ ying.yi@msa.hinet.net
}

Received 13 July 2015; accepted 19 August 2015; published 26 August 2015

\begin{abstract}
Due to the rise of biological and MEMS technology in recent years, some micro flow system components have drawn attention and been developed by many investigators. The importance of micro-pumps manufactured is higher than the other part of micro flow system since it is the power source of the entire micro-flow system and responsible for driving working fluid in the microfluidic system. In actual operation, the instability and bad dynamic characteristics of the micro-pump will cause larger fluid flow mobility error, such as transport behavior and response procedures failure, etc., and even damage the microfluidic system. Therefore, to investigate the stability and dynamic characteristics of a micro pump is necessary. The Finite element analysis (FEA), ANSYS Workbench, is employed to analyze the dynamic characteristics of this micro pump, and experiment is also considered in this study.
\end{abstract}

\section{Keywords}

Component, Micro Pump, Finite Element Analysis, Piezoelectric

\section{Introduction}

A micro fluid system driven by fluid system, like the human circulatory system, is the driving force source of the entire micro fluid flow system. And its feature is that it can a precision delivery of micro liquidity body, such as micro-pumps, micro valves and micro-sensors in the chip to conduct the biochemical reactions, separation and detection, and to increase the fuel injection efficiency by micro-pump delivery. At recently, miniaturization component is very widely applied in high precision industry, so miniature pumps will be a key component of indispensable.

Conducting research objective prediction, the micro fluid system future is bound to widespread use of its fluid delivery. This system will be through the high-performance micro-pumps provide impetus and accurate delivery flow, so the micro-flow system is also worthy to study. The micro-mechanical piezoelectric pumps are focused 
to investigate in this work. A diagram of this micro-mechanical piezoelectric pump is In this figure, a thin film, like as the cantilever mechanism, to drive the inlet and outlet valves, and its stability and dynamic characteristics affect the fluid flow accuracy. So to understand the micro pump actuation mechanism and exclude the actuation of the adverse factors, to increase the fluid current accuracy of control and precision density, and the other by understanding, of the future of energy design a decrease unstable phenomena institutions to serve the material and reduce processing costs, improve the overall Precision machining technology is the aim of this study. For the micro pump system study, Spencer et al. [1] investigated the piezoelectric film pump, membrane pump, subsequent research on thin-film micro pump driven pneumatic as Pol et al. [2] were found as Figure 1. Some researchers as Zengerle, etc. [3], W. Zhang et al. [4], Stemme et al. [5] and Benard et al. [6], they designed different types of micro-pumps, their operating conditions and performance made a big difference. Some studies turned to their attentions to the micro pneumatic pump, such as [7]-[10]. The outlet valve will dominate the dynamic characteristics of a micro pump. Therefore, the outlet valve is focused to investigate in this micro pump. To simplify, a cantilever beam type is employed to simulate the outlet value of a micro pump, as [11].

This outlet valve of a micro pump is also selected to study in this work. The finite element method, FEM, is employed to analyze the dynamic characteristics of a piezoelectric micro pump. An experimental analysis is also used to identify the dynamic properties accuracy.

\section{Finite Element Analysis}

The micro pump, Microjet PS31U5 piezoelectric micro pump, is employed to study. According to the literature published [13], the valves material of a micro pump, PDMS material i.e. Poly Dimethyl Siloxane, is used to study in this work. Table 1 shows the material parameters for this PDMS. Analysis software ANSYS Workbench is employed to study the dynamic characteristics of a micro pump. Element type Solid 187 is considered in this investigation to simulate the complex micro pump outlet valves. It is the three-dimensional 10-node entities, each node has $\mathrm{x}, \mathrm{y}, \mathrm{z}$ direction of displacement of the three degrees of freedom, elements have secondary displacement for irregular grid. This element has plasticity, super-elastic stress enhancement, large deformation, large strain characteristics, can also reduce free mesh problems caused by poor accuracy.

The material parameters, such as Young's modulus, Poisson's ratio and density, are set as a polydimethylsiloxane (PDMS) for this micro pump valves. Then, these elements will be meshed into very small elements. This mesh process must note the size and shape relations. After generating the grid produced, the boundary conditions of the micro pump valve have been set in this work. Finally, the finite element model can be to analyze and its results must be compared, verify its accuracy and converge analysis. Conversely, if the error is too large, it is necessary to return to pre-treatment part and to check material parameters or boundary conditions, and then reanalyzed.

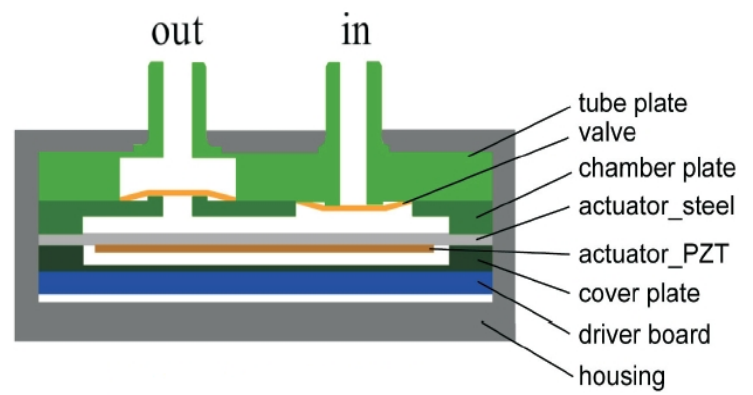

Figure 1. A diagram of this micro-mechanical piezoelectric pump [12].

Table 1. Material parameters for PDMS [13].

\begin{tabular}{cc}
\hline Material parameters & Unit \\
\hline Young's modulus & $1.53 \mathrm{MPa}$ \\
Density & $1051.3 \mathrm{~kg} / \mathrm{m}^{3}$ \\
Poisson ratio & 0.49 \\
\hline
\end{tabular}




\section{Discussion and Results}

This paper studies the dynamic characteristics of piezoelectric micro pump. By using the finite element analysis software ANSYS Workbench, the natural properties, dynamic characteristics of a micro pump can be studied. To make the results accuracy, experimental analysis is also considered in this work.

\subsection{Finite Element Analysis}

First, the convergence of results is necessary in finite element analysis. Figure 2 shows the convergence of the first natural frequency for a micro pump. If the number of elements meshed is over than 4463, the first natural frequency will be converged to $18 \mathrm{~Hz}$. Therefore, the 5000 elements meshed are selected to solve in this finite element analysis for this micro pump outlet valve. After the finite element analysis, the dynamic characteristics of micro pump valve can be observed. The natural frequencies and its corresponding mode shapes are found in Figure 3 displays the 1st natural frequency and mode shape in the color contour and displacement vector. In this figure, the largest vibration displacement occurs at the center of valve. That the branches of valve have the largest vibration in out of plane direction at $35.7 \mathrm{~Hz}$ is found in second mode. For same reason, it is found that the branches of valve have the largest vibration in plane direction at $35.8 \mathrm{~Hz}$. The torsion mode of the branches of valve can be found. Table 2 shows the natural frequencies of a micro pump valve.

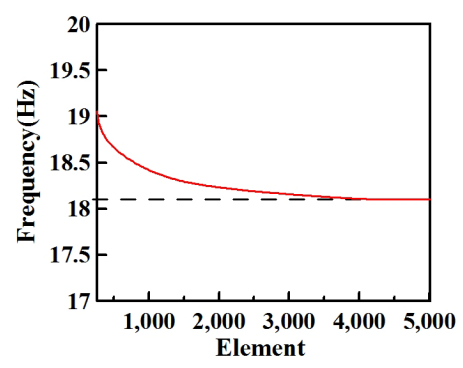

Figure 2. The convergence of the first natural frequency of a micro pump outlet valve.

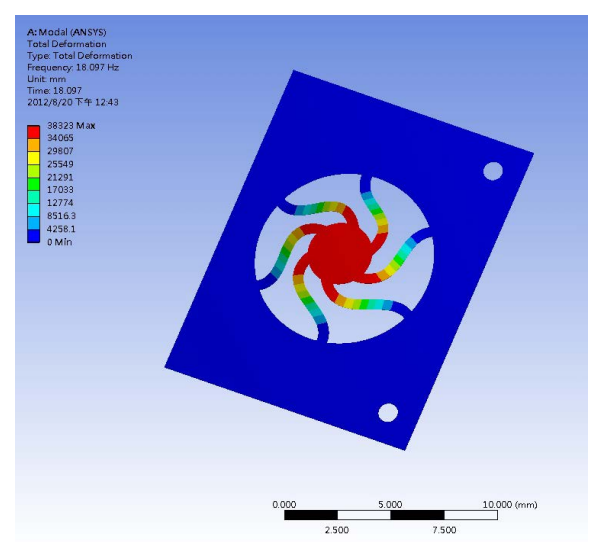

(a)

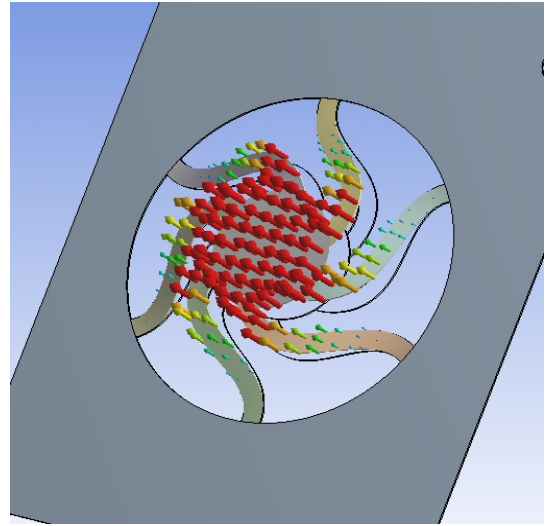

(b)

Figure 3. The 1st mode shape at $18.1 \mathrm{~Hz}$. (a) Color contour; (b) Displacement vector.

Table 2. Natural frequency of a micro pump valve.

\begin{tabular}{cc}
\hline Mode & Natural frequency (Hz) \\
\hline 1 & 18.1 \\
2 & 35.74 \\
3 & 35.79 \\
4 & 77.04 \\
\hline
\end{tabular}




\subsection{Experimental Analysis}

The experimental analysis is also considered in this work. An experimental setup is displayed in Figure 4. In this setup, a Laser displacement meter is employed to measure the vibration of the piezoelectric actuator and outlet valve of a micro pump. Figure 5 shows the time response of a piezoelectric actuator of a micro pump. In this figure, the perfect harmonic waves in this time response are found. This wave is excited by the piezoelectric actuator in a micro pump. Both the vibration waves of the piezoelectric actuator and outlet valve in a micro pump are considered to compare. Figure 6 illustrates the time response of an outlet valve of a micro pump. Because the residual vibration of is found as the outlet valve is operating. Therefore, the no perfect harmonic waves of the outlet valve when the piezoelectric actuator excites. However, both the frequencies of waves of the outlet valve and piezoelectric actuator are identical.

Frequency response is also considered in this work. Figure 7 shows the frequency response of a piezoelectric actuator of a micro pump. In this figure, the lowest vibration frequency of the piezoelectric actuator is $28 \mathrm{~Hz}$ when this actuator is excited. For the outlet valve, the frequency response is also considered as shown in Figure 8. From this figure, the vibration frequency of the outlet valve is also $28 \mathrm{~Hz}$. For both the piezoelectric actuator and outlet valve, the lowest vibration frequencies are identical when the actuator is excited.

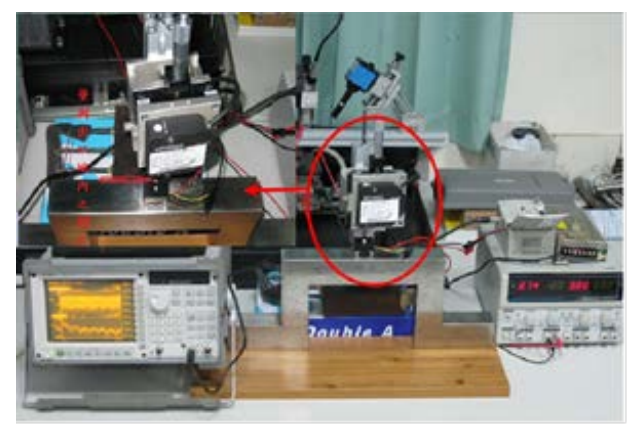

Figure 4. A photo of the experimental setup.

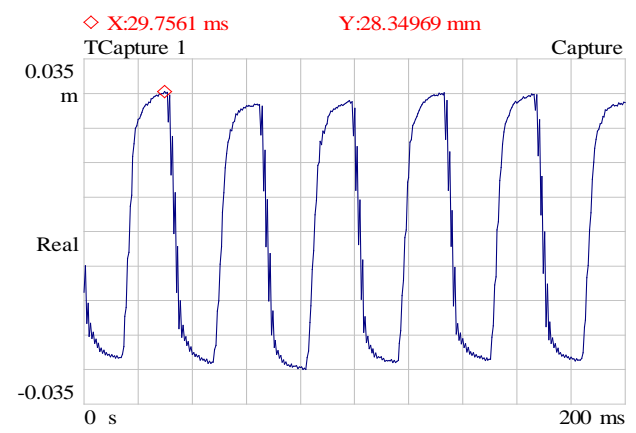

Figure 5. Time response of apiezoelectric actuator of a micro pump.

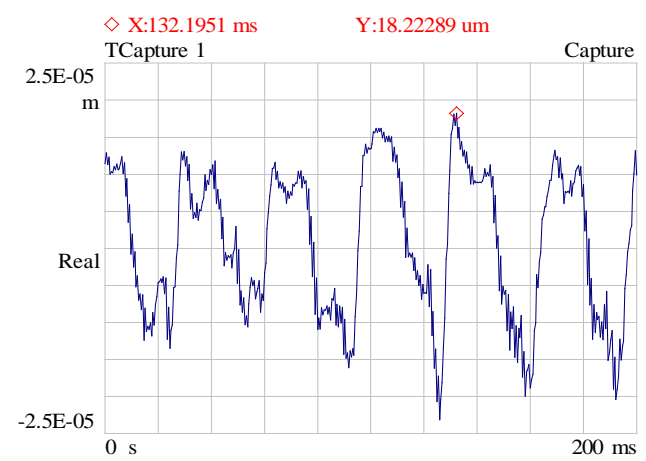

Figure 6. Time response of an outlet valve of a micro pump. 


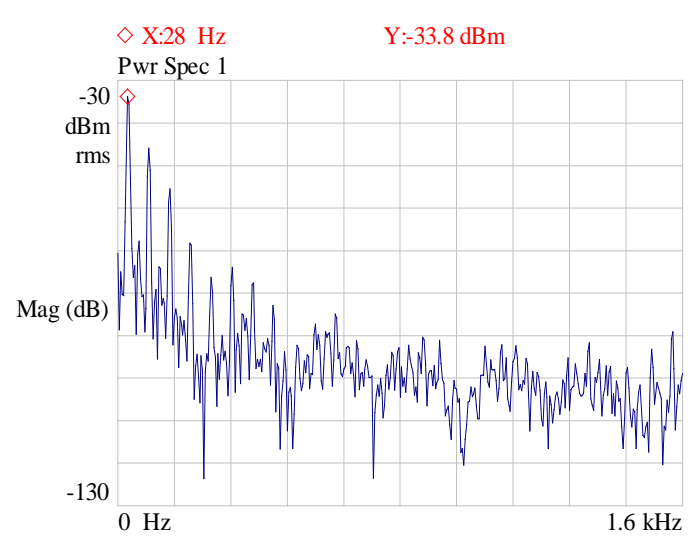

Figure 7. Frequency response of apiezoelectric actuator of a micro pump.

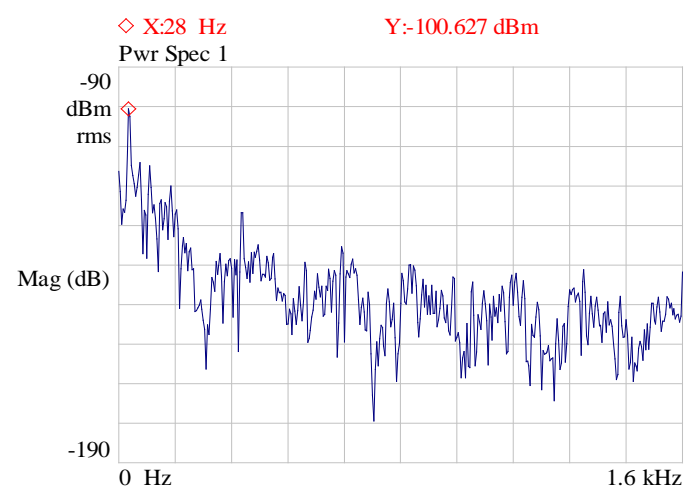

Figure 8. Frequency response of an outlet valve of a micro pump.

\section{Conclusions}

The natural properties of a piezoelectric micro pump have been investigated. The following conclusions can be drawn from this study.

1) Results show that the finite element analysis can be employed to study the natural properties of a micro pump.

2) Because the simulated PDMS is softer than the actual outlet value, the lowest natural frequency of the simulation is lower than the experiment.

3) Although the time responses of the actuator and outlet valve match very well, that the vibration frequency of the actuator is same as the outlet valve in a micro pump when the actuator is excited is found.

\section{Acknowledgements}

The authors would like to thank the National Science Council, Taiwan, for financially supporting this research through Grant NSC 102-2632-E-230-001-MY3.

\section{References}

[1] Spencer, W.J., Corbett, W.T., Dominguez, L.R. and Shafer, B.D. (1978) An Electronically Controlled Piezoelectric Insulin Pump and Valves. IEEE Trans. Sonics Ultrason, 25, 153-167. http://dx.doi.org/10.1109/T-SU.1978.31006

[2] Van de Pol, F.C.M., Van Lintel, H.T.G., Elwenspoek, M. and Fluitman, J.H.J. (1990) Thermopneumatic Micro Pump Based on Micro-Engineering Techniques. Sensors and Actuators, A: Physical, 21, 198-202. http://dx.doi.org/10.1016/0924-4247(90)85038-6

[3] Zengerle, R., Ulrich, J., Kluge, S., Richter, M. and Richter, A. (1995) A Bidirectional Silicon Micropump. Sensors and Actuators, A: Physical, 50, 81-86. http://dx.doi.org/10.1016/0924-4247(96)80088-4

[4] Zhang, W. and Ahn, C.H. (1996) A Bi-Directional Magnetic Micropump on a Silicon Wafer. Proceedings of the IEEE 
Solid-State Sensor and Actuator Workshop, Hilton Head Island, SC, June 1996, 94-97.

[5] Stemme, E. and Stemme, G. (1993) Valveless Diffuser/Nozzle Based Fluid Pump. Sensors and Actuators, A: Physical, 39, 159-167. http://dx.doi.org/10.1016/0924-4247(93)80213-Z

[6] Benard, W.L., Kahn, H., Heuer, A.H. and Huff, M.A. (1997) A Titanium-Nickel Shape Memory Alloy Actuated Micropump. Proceedings of the IEEE Solid-State Sensor and Actuator Workshop, Chicago, June 1997, 361-364.

[7] Vieider, C., Ohman, O. and Elderstig, H. (1995) A Pneumatically Actuated Micro Valve with a Silicone Rubber Membrane for Integration with Fluid-Handling Systems. Proceedings of the 8th International Conference on Solid-State Sensors and Actuators and Eurosensors IX, 2, June 1995, 284-286. http://dx.doi.org/10.1109/SENSOR.1995.721801

[8] Huff, M.A., Gilbert, J. and Schmidt, M.A. (1993) Flow Characteristics of a Pressure-Balanced Microvalve. Proceedings of the 7th International Conference on Solid State Actuators, Yokohama, June 1993, 98-101.

[9] Yanagisawa, K., Kuwano, H. and Tago, A. (1995) Electromagnetically Driven Microvalve. Microsystem Technologies, 2, 22-25. http://dx.doi.org/10.1007/BF02739524

[10] Jerman, H. (1994) Electrically-Activated, Normally-Closed Diaphragm Valve. Journal of Micromechanics and Microengineering, 4, 210-216. http://dx.doi.org/10.1088/0960-1317/4/4/006

[11] Olsson, A., Enoksson, P., Stemme, G. and Stemme, E. (1996) A Valveless Planar Pump Isotropically Etched in Silicon. Journal of Micromechanics and Microengineering, 6, 87-91. http://dx.doi.org/10.1088/0960-1317/6/1/020

[12] Microjet Technology Co., Ltd. (2007) CurieJet-Micro Pump. In: Product Type PS31U5.

[13] Lin, L.C. (2005) Numerical Analysis of Piezoelectric Micro Pump. M.S. Thesis, National Taiwan University, Taipei. 